\title{
Le nefropatie tubulo-interstiziali a trasmissione autosomica dominante (ADTKD)
}

\author{
Marco Quaglia ${ }^{1,2}$, Michele Battista ${ }^{1,2}$, Guido Merlotti, ${ }^{1,2}$, Martina Mazzariol ${ }^{1,2}$, Vincenzo Cantaluppi ${ }^{1,2}$ \\ ${ }^{1}$ S.C.D.U. di Nefrologia e Trapianto, A.O.U. "Maggiore della Carità" di Novara, Novara \\ ${ }^{2}$ Dipartimento di Medicina Traslazionale, Università del Piemonte Orientale, Novara
}

\begin{abstract}
Autosomal dominant tubulointerstitial kidney disease (ADTKD)
Autosomal Dominant Tubulointerstitial Kidney Diseases (ADTKD) are a group of autosomal dominant hereditary nephropathies which share a rather aspecific pathological picture of interstitial fibrosis and tubular atrophy (IFTA) and a slowly progressing tubulointerstitial clinical profile: end-stage renal disease (ESRD) generally occurs between 30 and 50 years.

Four types of ADTKD have been described so far, each showing some peculiarities: hepatocyte nuclear factor-1 $\beta$ (HNF1 $\beta$ ) ADTKD, in which ADTKD can be associated with renal cysts and a wide spectrum of congenital abnormalities of kidney and urinary tract (CAKUT) and extra-renal manifestations (diabetes, liver enzyme alterations, genital malformations), uromodulin (UMOD) ADTKD, characterised by early onset of hyperuricemia and gout, mucin-1 (MUC-1) ADTKD, a recently discovered form which appears to be an isolated tubulointerstitial nephropathy, and renin (REN) ADTKD, which appears in childhood and is characterised by anemia and mild signs of hyporeninism. A better awareness is needed in order to perform genetic analysis whenever clinical setting is compatible with this nephropathy. A timely diagnosis can have an important impact on both the patient and his family members in terms of therapy and prognosis, also at the prospect of renal transplant.
\end{abstract}

Keywords: Autosomal dominant tubulointerstitial kidney disease, Genetic analysis, Genetic mutations, Hepatocyte nuclear factor-1 $\beta$, Mucin-1, Renin, Uromodulin

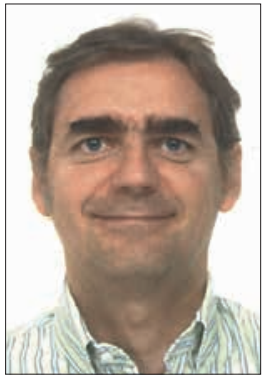

Marco Quaglia

\section{Introduzione}

Le nefropatie tubulo-interstiziali ereditarie a trasmissione autosomica dominante (ADTKD)

Si tratta di un gruppo di nefropatie accomunate da una trasmissione autosomica dominante (AD), da un quadro istologico in genere aspecifico, caratterizzato da atrofia tubulare e fibrosi interstiziale (IFTA), con immunofluorescenza

Accepted: April 18, 2017

Published online: August 3, 2017

Indirizzo per la corrispondenza:

Dr. Marco Quaglia

Dipartimento di Medicina Traslazionale

Unità di Nefrologia e Trapianto

Università del Piemonte Orientale

Via Solaroli, 17

28100 Novara

marco.quaglia@gmail.com

negativa per immunoglobuline e frazioni del complemento, e da un profilo clinico nefrologico tubulo-interstiziale che determina una CKD in genere lentamente progressiva verso I'insufficienza renale cronica terminale (ESRD). Questa si verifica in genere nella fascia tra i 30 e i 50 anni (età media intorno ai 45 anni), sia pur con un'ampia variabilità inter e intra-familiare (da 17 a oltre 70 anni) (1-3).

In una recente Consensus Conference KDIGO sono state identificate 4 forme principali di ADTKD, causate da altrettante mutazioni genetiche e una forma "non-altrimenti specificata" (non-otherwise specified: NOS) che raggruppa forme ascrivibili a mutazioni di altri geni non ancora identificate (4):

- $\quad$ ADTKD da mutazioni del gene TCF2 codificante per l'Hepatocyte nuclear factor $B$ (HNF1 $\beta$ ): HNF1 $\beta$-ADTKD;

- ADTKD da mutazioni del gene codificante per l'Uromodulina: UMOD-ADTKD;

- $\quad$ ADTKD da mutazioni del gene codificante per la Mucina-1: MUC1-ADTKD;

- $\quad$ ADTKD da mutazioni del gene codificante per il precursore della Renina: REN-ADTKD;

- $\quad$ ADTKD da mutazioni non-altrimenti specificate: NOSADTKD. 
Oltre alle manifestazioni cliniche tipiche delle nefropatie tubulo-interstiziali (sedimento urinario aspecifico, proteinuria modesta o assente, assenza di ipertensione arteriosa nelle fasi iniziali, nicturia o enuresi nei bambini), alcune forme, in particolare HNF1 $\beta$-ADTKD $(5,6)$, sono caratterizzate da peculiari manifestazioni sistemiche extrarenali che possono indirizzare verso la diagnosi differenziale (Tab. I).

\section{Analisi delle forme di ADTKD}

\section{1) ADTKD - HNF16}

Questa nefropatia si associa spesso a manifestazioni pleomorfe, sia a livello renale che extrarenale $(5,6)$ in accordo con il complesso ruolo esercitato dal fattore di trascrizione HNF1 $\beta$ nell'embriogenesi di molti organi tra cui reni, fegato, pancreas e apparato genitale (7). Mutazioni di HNF1 $\beta$ si riscontrano nel $10-30 \%$ delle forme CAKUT, che, a loro volta, rappresentano il tipo più frequente di disordine dello sviluppo identificato nel periodo pre-natale (8). In una nostra casistica di pazienti nefrologici e portatori di trapianto renale abbiamo riscontrato una prevalenza del $9 \%$ adottando come criterio di screening la presenza di una nefropatia tubulointerstiziale a trasmissione $A D$ in associazione con la presenza di una qualunque anomalia strutturale renale (RSA) (9).

HNF1 $\beta$ regola anche la trascrizione di vari geni codificanti per proteine del cilio primario e anche del gene codificante per UMOD, per cui un'alterata funzione di questo fattore di trascrizione determina una profonda riduzione dell'espressione di geni codificanti per cistoproteine (Pkhd1, Umod, Pkd2), promuovendo la cistogenesi (7).
ADTKD-HNF1 $\beta$ include infatti diversi quadri renali caratterizzati sia da cisti renali (in genere in numero inferiore a 5 per rene e non deformanti il profilo renale, a differenza delle cisti dell'ADPKD) che da un'ampia gamma di aspetti malformativi, tra i quali i più frequenti sono rappresentati da:

- reni multicistici displasici (MCDK)

- reni ipoplasici o displasici

- agenesia renale (monorene)

- rene ectopico

- reflusso vesico-ureterale (VUR)

- idronefrosi

Alcune di queste alterazioni possono essere presenti già in epoca pre-natale, quando i reni possono avere un aspetto iperecogeno conseguente alla presenza di multiple microcisti, displasia renale o dilatazione tubulare.

Altre alterazioni sono costituite dalla malattia renale glomerulocistica ipoplasica familiare, dall'oligomeganefronia e dal rene a ferro di cavallo. Per contro è anche possibile che $i$ reni siano morfologicamente del tutto normali.

Dal punto di vista laboratoristico, è spesso presente una tubulopatia che determina un wasting di magnesio $(10,11)$ e potassio, con conseguenti ipomagnesiemia e ipopotassiemia, e, talora, un'iperuricemia ipouricosurica (forse secondaria a una ridotta espressione del gene codificante per UMOD, il cui promoter è attivato da HNF1 $\beta$ ).

Le principali manifestazioni extra-renali di ADTKD-HNF1 $\beta$ sono $(5,8)$ :

- diabete mellito a insorgenza precoce

- malformazioni dell'apparato genitale, soprattutto nella

TABELLA I - Principali caratteristiche delle ADTKD

\begin{tabular}{|c|c|c|c|c|}
\hline & HNF1 $\beta$ & UMOD & MUC1 & REN \\
\hline Fisiopatologia & $\begin{array}{l}\text { Alterata embriogenesi a carico di vari } \\
\text { organi }\end{array}$ & $\begin{array}{l}\text { Deposizione di } \\
\text { UMOD mutata nelle } \\
\text { cellule della TAL }\end{array}$ & $\begin{array}{l}\text { Deposizione di MUC1 } \\
\text { mutata nelle cellule di } \\
\text { TAL e DCD }\end{array}$ & $\begin{array}{l}\text { Deposizione di pre-prorenina } \\
\text { mutata nelle cellule dell'appa- } \\
\text { rato iuxtaglomerulare }\end{array}$ \\
\hline Gotta & Possibile & $\begin{array}{l}\text { Frequente, può esor- } \\
\text { dire nell'infanzia }\end{array}$ & Solo in CKD avanzata & $\begin{array}{l}\text { Frequente, può esordire nell'in- } \\
\text { fanzia }\end{array}$ \\
\hline Laboratorio & $\begin{array}{l}\text { Ipomagnesiemia, } \\
\text { ipokaliemia, iperuricemia, } \\
\text { alterazione enzimi epatici }\end{array}$ & $\begin{array}{l}\text { Iperuricemia ipou- } \\
\text { ricosurica (FE acido } \\
\text { urico }<5 \% \text { ) }\end{array}$ & Nessuna & $\begin{array}{l}\text { Iperuricemia ipouricosurica, } \\
\text { iperkaliemia, } \\
\text { anemia nell'infanzia }\end{array}$ \\
\hline
\end{tabular}

*Età media e range si riferiscono alla casistica di Bleyer et al. (1). 
femmina (p. es., utero bicorne o didelfo) ma anche nel maschio (p. es., assenza congenita dei vasi deferenti e alterazioni delle vescicole seminali con infertilità) (12)

- anomalie degli enzimi epatici (citolisi e colestasi), in genere lievi.

Sono stati inoltre segnalati: iperparatiroidismo primitivo, sordità neurosensoriale, carcinoma renale a cellule cromofobe, epilessia, autismo e ritardo mentale (5).

Il diabete rappresenta la manifestazione più frequente. La mutazioni di HNF1 $\beta$ sono una rara causa di MODY (Maturity Onset Diabetes of the Young) di tipo 5 ( $<2 \%$ dei casi, vs il $60 \%$ attribuito a HNF1 $\alpha$ ), che si manifesta tipicamente prima dei 25 anni, mentre una forma di diabete di tipo 2 compare in circa metà dei pazienti tra la terza e la quarta decade. Sono state descritte forme di ipoplasia pancreatica e anche di disfunzione subclinica del pancreas esocrino $(5,8)$.

Un aspetto rilevante nella gestione di questi pazienti è costituito dalla predisposizione a un'aumentata insorgenza di diabete post-trapianto (PTDM). L'inibitore delle calcineurine (CNI) potrebbe giocare un ruolo particolare nello slatentizzare questa forma di diabete attraverso un'inibizione dose-dipendente della trascrizione dell'allele sano di HNF1 $\beta$. Questi dati, se confermati, potrebbero fornire un'indicazione ad adottare protocolli di minimizzazione del CNI in questi pazienti (13).

La complessità dello spettro di manifestazioni ha indotto alcuni Autori a formulare modelli predittivi attraverso l'attribuzione di score ai vari parametri (tra i quali la presenza di iperecogenicità parenchimale, cisti renali, anomalie genitali e pancreatiche) per valutare l'indicazione all'analisi genetica nei pazienti con manifestazioni sospette per ADTKD-HNF1 3 . Il modello di Faguer $\mathrm{S}$. et al. ha un profilo che lo rende uno strumento interessante per lo screening (sensibilità 98.2\%; specificità 41.1\%) (14). Raaijmakers A. et al. hanno invece impiegato un sistema basato sulla valutazione di alcuni criteri clinico-laboratoristici renali ed extrarenali, sia personali che familiari, identificando nella presenza di cisti renali di origine ignota e nell'ipomagnesiemia gli elementi più fortemente predittivi di questa forma (15).

\section{2) $A D T K D-U M O D$}

Questa denominazione sostituisce le vecchie denominazioni di nefropatia iperuricemica familiare e malattia cistica della midollare di tipo 2. Quest'ultimo termine in particolare era fuorviante (2), in quanto né le microcisti tubulari né cisti di maggiori dimensioni rilevabili ecograficamente né la loro collocazione in sede midollare sono caratteristiche patognomoniche. Sono state descritte finora circa $2000 \mathrm{fa}$ miglie con ADTKD-UMOD $(1-4,16,17)$.

UMOD (o proteina di Tamm-Horsfall) rappresenta la proteina fisiologicamente più abbondante nelle urine. Secreta esclusivamente dalle cellule tubulari della branca spessa ascendente (TAL) dell'ansa di Henle attraverso un meccanismo di clivaggio proteolitico, ha complesse funzioni solo in parte note, tra le quali un ruolo importante nel mantenere l'impermeabilità della TAL all'acqua e nel facilitare il trafficking del cotrasportatore NKCC2 sulla superficie della cellula tubulare e la sua attività, oltre ad avere un effetto anti-batterico e di inibizione della cristallizzazione (16-19). Inoltre, alcuni polimorfismi genetici di UMOD conferirebbero un'aumentata suscettibilità allo sviluppo di CKD e ipertensione arteriosa, forse in relazione con un'iperattività di NKCC2 con eccessivo riassorbimento sodico a livello della TAL (20); inoltre livelli urinari elevati di UMOD (UUMOD) sono stati associati a outcome renali migliori e a una riduzione della mortalità cardiovascolare (21).

La fisiopatologia di questa ADTKD-UMOD è riconducibile alla produzione di una forma mutata di UMOD (circa metà delle mutazioni di UMOD descritte determina la delezione di un residuo di cisteina che impedisce alla proteina di conformarsi correttamente), che ne condiziona una deposizione anomala nel reticolo endoplasmatico delle cellule tubulari della TAL dell'ansa di Henle $(1,4)$. Questo accumulo intracellulare determina una disfunzione e quindi un'apoptosi della cellula tubulare (atrofia tubulare). La proteina mutata, inoltre, potrebbe raggiungere l'interstizio renale attraverso la membrana baso-laterale della cellula tubulare e innescare processi di flogosi e quindi di fibrosi interstiziale $(3,19)$.

Infine, il mancato trasporto sulla membrana apicale della proteina codificata dall'allele mutato determina una riduzione delle funzioni di UMOD, con conseguente ridotte espressione e attività di NKCC2, aumento dell'escrezione sodica (per ridotto riassorbimento nella TAL), lieve deplezione dei volumi e aumentato riassorbimento di sodio e acido urico a livello del tubulo contorto prossimale. L'iperuricemia uricosurica che ne consegue (con escrezione frazionale dell'acido urico in genere inferiore al 5\%) determina spesso una gotta, che può comparire già nella tarda adolescenza (17). Sia l'iperuricemia che la gotta possono precedere la comparsa dell' insufficienza renale cronica, che conduce a ESRD tra i 30 e i 70 anni, mediamente intorno ai 50 anni $(1,4)$.

La terapia con ipouricemizzanti (allopurinolo e febuxostat) è in grado di prevenire l'insorgenza della gotta se assunta in giovane età, mentre un suo effetto di rallentamento della progressione della CKD è controverso. L'allopurinolo è teratogeno e deve essere quindi sospeso prima dell'avvio di una gravidanza (18).

\section{3) ADTKD-MUC1}

Questa denominazione sostituisce la vecchia espressione "malattia cistica della midollare di tipo 2" (2). Sono state descritte finora circa 1000 famiglie con ADTKD-MUC1 $(1,4)$. Analogamente a quanto descritto per ADTKD-UMOD, anche in questa forma il meccanismo fisiopatologico è riconducibile alla produzione e alla deposizione di una proteina mutata 
(costituita da un peptide "frameshift" carico positivamente, prodotto come conseguenza dell'inserzione di una citosina che altera una sequenza ripetuta di 20 aminoacidi nel domain extracellulare della Mucina-1) a livello del reticolo endoplasmatico delle cellule tubulari della TAL dell'ansa di Henle, del tubulo distale e del dotto collettore, causandone l'apoptosi $(22,23)$.

La Mucina-1 è una mucoproteina ancorata alla membrana cellulare, ampiamente espressa in sedi extra-renali, in particolare nelle cellule epiteliali di mammella, polmone, tratto gastroenterico e ghiandole sebacee della cute, con complesse funzioni sia di rivestimento protettivo (domain extracellulare glicosilato) che di signaling intracellulare (domain intracellulare). Un'aumentata espressione di MUC1 è stata riscontrata nell'adenocarcinoma del dotto pancreatico e nelle neoplasie biliari e si associa a una prognosi peggiore (24). Le mutazioni di MUC1 possono quindi essere coinvolte in importanti ambiti extra-renali; tuttavia, nonostante la deposizione della proteina mutata avvenga in molti tessuti, i pazienti con ADTKD-MUC1 non sembrano avere manifestazioni extra-renali: in particolare, non è dimostrata un'aumentata incidenza di neoplasie e il quadro clinico sembra limitato alla nefropatia tubulo-interstiziale cronica.

L'insorgenza di ESRD avviene in metà dei pazienti tra i 30 e i 50 anni, in media intorno ai 43 anni. È da segnalare che alcuni pazienti sviluppano una progressione rapida $(-7 \mathrm{~mL} / \mathrm{min} /$ anno) dopo riduzione del GFR al di sotto di $30 \mathrm{~mL} / \mathrm{min}$, che determina la comparsa di un'ESRD più precoce, intorno ai 30 anni $(1,4)$. Nella nostra casistica i pazienti con ADTKD-MUC1 tendono a progredire verso I'ESRD più rapidamente che nelle altre forme di ADTKD (23). Cisti renali sono presenti in circa la metà dei pazienti e non sono mai in sede midollare (a conferma dell'inadeguatezza della precedente denominazione di malattia cistica della midollare).

Non esiste una terapia specifica.

\section{4) ADTKD-REN}

Questa forma è decisamente più rara delle tre precedenti, essendo stata descritta finora in meno di 20 famiglie con $\operatorname{ADTKD}(1,4)$.

Analogamente a quanto descritto per ADTKD-UMOD e ADTKD-MUC1, anche in questa forma il meccanismo fisiopatologico è riconducibile all'accumulo di una proteina mutata: mutazioni del gene REN che codifica per il precursore della renina (pre-prorenina) determinano la produzione di una proteina che si deposita non solo nelle cellule dell'apparato iuxtaglomerulare ma anche in quelle delle cellule di vari segmenti tubulari (nei quali il gene REN è normalmente espresso), condizionandone una disfunzione e infine l'apoptosi cellulare.

Il quadro clinico esordisce nell'infanzia ed è caratterizzato da anemia iporigenerativa secondaria a ridotta sintesi di eritropoietina (che precede l'insorgenza di insufficienza renale e tipicamente si risolve nell'adolescenza, in concomitanza con l'aumento della sintesi di testosterone), poliuria (enuresi) e sintomi clinici di lieve iporeninismo: lieve ipotensione arteriosa, tendenza all'iperkaliemia e predisposizione a sviluppare AKI in corso di eventi favorenti (p. es., disidratazione secondaria a stati febbrili) a causa della lieve contrazione cronica dei volumi, che è anche responsabile dell'iperuricemia ipouricosurica con possibile gotta a esordio nell'adolescenza.

La terapia specifica di questa forma include la somministrazione di eritropoietina, il mantenimento di una dieta ad alto contenuto sodico ed eventualmente la somministrazione di fludrocortisone, che corregge l'ipoattività del sistema renina-angiotensina e potrebbe avere un effetto inibitorio sull'espressione del gene mutato (25-27).

\section{Diagnosi di ADTKD}

I criteri per sospettare la diagnosi di una di queste forme sono:

1. una storia familiare di nefropatia con profilo clinico tubulo-interstiziale, a trasmissione AD oppure

2. in assenza di familiarità, evidenza di un quadro istologico compatibile oppure di manifestazioni extra-renali compatibili con HNF1 $\beta$ oppure di una storia di iperuricemia/ gotta a esordio precoce.

I criteri per stabilire una diagnosi certa di ADTKD sono:

1. una storia familiare di nefropatia con profilo clinico tubulo-interstiziale a trasmissione $A D$ e un quadro istologico compatibile in almeno 1 membro della famiglia oppure

2. dimostrazione della presenza di una mutazione in uno dei 4 geni nel probando o almeno in uno dei membri della famiglia.

\section{Impatto clinico della diagnosi di ADTKD}

Pervenire a una diagnosi certa di ADTKD ha un importante impatto clinico sia sul paziente che sui suoi familiari per diverse ragioni:

1. molti di questi pazienti rappresentano degli ottimi candidati al trapianto renale per le loro caratteristiche cliniche (ESRD in età relativamente giovane, spesso in assenza di significative comorbidità, con l'eccezione di alcune forme di ADTKD-HNF1 $\beta$ associate a diabete mellito; nessun rischio di recidiva della nefropatia sul rene trapiantato). Qualora altri membri della famiglia esprimano la loro disponibilità a una donazione di rene, una diagnosi certa di ADTKD impone che questi parenti apparentemente sani si sottopongano all'analisi genetica per escludere che siano invece anche loro affetti dalla medesima nefropatia. È da sottolineare che, soprattutto in alcune forme lievi 
di ADTKD-MUC1 e ADTKD-UMOD (28), i pazienti affetti possono avere una funzione renale conservata e un quadro nefrologico silente anche nella terza decade di età (sedimento urinario indifferente, assenza di proteinuria e di ipertensione arteriosa, reni ecograficamente normali, senza alcuna cisti) e anche le manifestazioni extra-renali che spesso accompagnano queste forme possono essere completamente assenti e non sono in ogni caso patognomoniche (per esempio, la gotta). Sono stati descritti casi di donazione da parte di membri della famiglia con ADTKD subclinica (29). Nella nostra esperienza la prevalenza di nefropatie su base genetica misconosciute nella popolazione dei pazienti pervenuti al trapianto renale senza una diagnosi istologica è pari al $4.3 \%$ e include prevalentemente forme di ADTKD-UMOD e HNF1 $\beta$ (30);

2. la diagnosi genetica di ADTKD evita l'eventuale ricorso a una biopsia renale in altri membri della famiglia che manifestino segni di nefropatia (la biopsia renale sarebbe scarsamente informativa essendo il quadro istologico aspecifico) $(1,3)$;

3. la diagnosi genetica di ADTKD è utile per gestire al meglio il follow-up del paziente e ottimizzare la gestione generale delle forme tubulo-interstiziali (impiegare con cautela il diuretico ed evitare la dieta iposodica per il rischio di esacerbare la deplezione di volume e l'iperuricemia, soprattutto nella ADTKD-UMOD e nella ADTKD-REN; evitare i FANS, soprattutto nella ADTKD-REN, per il rischio di precipitare un'insufficienza renale acuta); utilizzare alcune terapie specifiche (fludrocortisone, dieta ad alto contenuto sodico ed eritropoietina nelle ADTKD-REN; allopurinolo nelle ADTKD-UMOD); eventuale trapianto combinato di rene e pancreas in presenza di MODY di tipo 5 $(1,13)$;

4. la diagnosi genetica di ADTKD in un probando può avere un rilevante impatto su molti membri della famiglia, attraverso più generazioni, in termini di consapevolezza della malattia e della sua prognosi;

5. infine i membri della famiglie affette possono contribuire alla ricerca qualora siano disposti a partecipare a studi volti a identificare nuove mutazioni responsabili di ADTKD e a chiarire i rapporti genotipo/fenotipo, i meccanismi fisiopatologici e altri aspetti di queste malattie rare.

\section{Conclusione}

In conclusione, la presenza di una nefropatia familiare con un profilo clinico tubulo-interstiziale, a trasmissione $A D$, con progressione all'ESRD mediamente intorno all'età di 45 anni, deve indurre a prendere in considerazione l'analisi genetica di queste quattro forme $(1,4)$. La presenza di alcuni segni o sintomi di malattia può indirizzare verso una forma specifica, consentendo una ricerca genetica mirata. La presenza di quadri malformativi renali (per esempio, reni displasici o ipoplasici) e di alcune manifestazioni extra-renali (per esempio, diabete mellito, malformazioni dell'apparato genitale) e di laboratorio (soprattutto ipomagnesiemia) indirizza prioritariamente verso una ADTKD-HNF1 $\beta$ (6) e alcuni score diagnostici sono stati recentemente proposti per guidare il ricorso all'analisi genetica nei casi sospetti $(14,15)$; una storia di iperuricemia o di gotta precoci, comparse molto prima dell'insufficienza renale, è suggestiva per ADTKD-UMOD (16); una nefropatia tubulo-interstiziale cronica isolata è compatibile con ADTKD-MUC1 (22); un quadro di anemia a esordio infantile accompagnato da segni lievi di iporeninismo, infine, può suggerire una forma di ADTKD-REN (26).

La diagnosi di queste forme è genetica, mentre la biopsia renale è scarsamente informativa in quanto dimostra in genere un quadro aspecifico di IFTA (3).

Una maggiore consapevolezza riguardo a queste forme è necessaria per ridurne la sotto-diagnosi, che ha importanti ricadute sia sul paziente che sui suoi familiari, anche nella prospettiva del trapianto renale.

\section{Disclosures}

Financial support: No financial support was received for this submission.

Conflict of interest: The authors have no conflict of interest.

\section{Bibliografia}

1. Bleyer AJ, Kidd K, Živná $M$, et al. Autosomal Dominant Tubulointerstitial Kidney Disease. Adv Chronic Kidney Dis. 2017;24(2):86-93.

2. Bleyer AJ, Kmoch S. Autosomal dominant tubulointerstitial kidney disease: of names and genes. Kidney Int. 2014;86(3): 459-461.

3. Ekici $A B$, Hackenbeck $T$, Morinière $V$, et al. Renal fibrosis is the common feature of autosomal dominant tubulointerstitial kidney diseases caused by mutations in mucin 1 or uromodulin. Kidney Int. 2014;86(3):589-599.

4. Eckardt KU, Alper SL, Antignac C, et al. Kidney Disease: Improving Global Outcomes Autosomal dominant tubulointerstitial kidney disease: diagnosis, classification, and management - A KDIGO consensus report. Kidney Int. 2015;88(4):676-683.

5. Bockenhauer D, Jaureguiberry G. HNF1B-associated clinical phenotypes: the kidney and beyond. Pediatr Nephrol. 2016; 31(5):707-714.

6. Clissold RL, Hamilton AJ, Hattersley AT, Ellard S, Bingham C. HNF1B-associated renal and extra-renal disease - an expanding clinical spectrum. Nat Rev Nephrol. 2015;11(2):102-112.

7. Faguer $S$, Decramer $S$, Devuyst $O$, et al. Expression of renal cystic genes in patients with HNF1B mutations. Nephron Clin Pract. 2012;120(2):c71-78.

8. Faguer $S$, Decramer $S$, Chassaing $N$, Bellanné-Chantelot $C$. Diagnosis, management, and prognosis of HNF1B nephropathy in adulthood. Kidney Int. 2011;80(7):768-776.

9. Musetti C, Quaglia M, Mellone S, Pagani A. Chronic renal failure of unknown origin is caused by HNF1B mutations in $9 \%$ of adult patients: a single centre cohort analysis. Nephrology. 2014;19(4):202-209.

10. van der Made $\mathrm{Cl}$, Hoorn EJ, de la Faille $\mathrm{R}$, Karaaslan $\mathrm{H}$. Hypomagnesemia as First Clinical Manifestation of ADTKDHNF1B: A Case Series and Literature Review. Am J Nephrol. 2015;42(1):85-90. 
11. MusettiC, Quaglia M, Stratta P, Giordano M. Hypomagnesemia and progressive chronic kidney disease: thinking of HNF1B and other genetic nephropathies. Kidney Int. 2015;88(3): 641.

12. Mieusset R, Fauquet I, Chauveau D, Monteil L. The spectrum of renal involvement in male patients with infertility related to excretory-system abnormalities: phenotypes, genotypes, and genetic counseling. J Nephrol. 2017;30(2):211-218.

13. Faguer S, Esposito L, Casemayou A, et al. Calcineurin Inhibitors Downregulate HNF-1 $\beta$ and May Affect the Outcome of HNF1B Patients After Renal Transplantation. Transplantation. 2016;100(9):1970-1978.

14. Faguer S, Chassaing N, Bandin F, et al. The HNF1B score is a simple tool to select patients for HNF1B gene analysis. Kidney Int. 2014;86(5):1007-1015.

15. Raaijmakers A, Corveleyn A, Devriendt K, van Tienoven TP. Criteria for HNF1B analysis in patients with congenital abnormalities of kidney and urinary tract. Nephrol Dial Transplant. 2015;30(5): 835-842.

16. Venkat-Raman G, Gast C, Marinaki A, Fairbanks L. From juvenile hyperuricaemia to dysfunctional uromodulin: an ongoing metamorphosis. Pediatr Nephrol. 2016;31(11):2035-2042.

17. Bollée $\mathrm{G}$, Dahan K, Flamant $\mathrm{M}$, et al. Phenotype and outcome in hereditary tubulointerstitial nephritis secondary to UMOD mutations. Clin J Am Soc Nephrol. 2011;6(10):2429-2438.

18. Gibson T. Hyperuricemia, gout and the kidney. Curr Opin Rheumatol. 2012;24(2):127-131.

19. Rampoldi L, Scolari F, Amoroso A, Ghiggeri G. The rediscovery of uromodulin (Tamm-Horsfall protein): from tubulointerstitial nephropathy to chronic kidney disease. Kidney Int. 2011; 80(4):338-347.

20. Scolari F, Izzi C, Ghiggeri GM. Uromodulin: from monogenic to multifactorial diseases. Nephrol Dial Transplant. 2015;30(8): 1250-1256.

21. Garimella PS, Biggs ML, Katz R, et al. Urinary uromodulin, kidney function, and cardiovascular disease in elderly adults.
Kidney Int. 2015;88(5):1126-1134.

22. Bleyer AJ, Kmoch S, Antignac $\mathrm{C}$, et al. Variable clinical presentation of an MUC1 mutation causing medullary cystic kidney disease type. Clin J Am Soc Nephrol. 2014;9(3):527-535.

23. Musetti C, Babu D, Fusco I, et al. Testing for the cytosine insertion in the VNTR of the MUC1 gene in a cohort of Italian patients with autosomal dominant tubulointerstitial kidney disease. J Nephrol 2016;29(3):451-455.

24. Moschovis D, Bamias G, Delladetsima I. Mucins in neoplasms of pancreas, ampulla of Vater and biliary system. World J Gastrointest Oncol. 2016;8(10):725-734.

25. Zivná $M$, Hůlková $H$, Matignon $M$, et al. Dominant renin gene mutations associated with early-onset hyperuricemia, anemia, and chronic kidney failure. Am J Hum Genet. 2009;85(2):204-213.

26. Bleyer AJ, Zivná M, Hulková H, Hodanová K. Clinical and molecular characterization of a family with a dominant renin gene mutation and response to treatment with fludrocortisone. Clin Nephrol. 2010;74(6):411-422.

27. Kmoch S, Živná M, Bleyer AJ. Autosomal Dominant Tubulointerstitial Kidney Disease, REN-Related. In: Pagon RA, Adam MP, Ardinger HH, Wallace SE, Amemiya A, Bean LJH, Bird TD, Ledbetter N, Mefford HC, Smith RJH, Stephens K, editors. Source GeneReviews ${ }^{\circledR}$ [Internet]. Seattle (WA): University of Washington, Seattle; 1993-2017. 2011 Apr 5 [updated 2015 Dec 29].

28. Smith GD, Robinson C, Stewart AP, Edwards EL. Characterization of a recurrent in-frame UMOD indel mutation causing lateonset autosomal dominant end-stage renal failure. Clin J Am Soc Nephrol. 2011;6(12):2766-2774.

29. Ross LF, Saal HM, David KL, Anderson RR. Technical report: Ethical and policy issues in genetic testing and screening of children. Genet Med. 2013;15(3):234-245.

30. Quaglia M, Musetti C, Ghiggeri GM, et al. Unexpectedly high prevalence of rare genetic disorders in kidney transplant recipients with an unknown causal nephropathy. Clin Transplant. 2014;28(9):995-1003. 